Lepr Rev (1997) 68, 195-200

\title{
Editorial
}

\section{WE NEED TO KNOW WHAT IS HAPPENING TO THE INCIDENCE OF LEPROSY}

\section{Why we need to know}

The current global strategy for leprosy is based on reducing the prevalence of the disease using short course multidrug therapy (MDT). The case for using prevalence as an indicator for the programme has been well argued in that it is easy to measure, responsive to change, and appropriate for a disease of long duration. However the argument may inadvertently imply that incidence is therefore not important, or certainly much less important. The prevalence of leprosy has dramatically fallen over the past 10 years (Figure 1) as a result of the global implementation of MDT although this now appears to be levelling off. ${ }^{1}$ Prevalence is now no longer such a useful indicator, we need to know what is happening to the incidence of leprosy.

Incidence rates give us vital information on the transmission of disease. We need to know the underlying incidence of leprosy to know whether any impact is being made on transmission. It seems likely that transmission is decreasing in many countries and has been for many decades, indeed long term trends are more convincing. ${ }^{2}$ At present the intense anti-leprosy activities in the major endemic countries make it difficult to assess the underlying trends in incidence in the countries where it matters the most. Knowledge of the trends in incidence would help in understanding which factors might have the greatest impact, chemotherapy, BCG or socio-economic changes. Information on the trends in incidence of leprosy is needed to plan for future anti-leprosy activities.

\section{Registered prevalence and true prevalence of leprosy}

Prevalence is the number of people with a disease at one point in time (point prevalence) or over a given period of time (period prevalence). The leprosy data usually presented is point prevalence, commonly at the end of the calendar year, but some programmes do use period prevalence. Actually, what is used is the number of patients registered for treatment which includes people who have been mis-diagnosed as leprosy, cured patients, and even patients who have died if the registers are not kept up to date. The registered prevalence excludes those who have leprosy but who have not been registered for treatment. The WHO global 


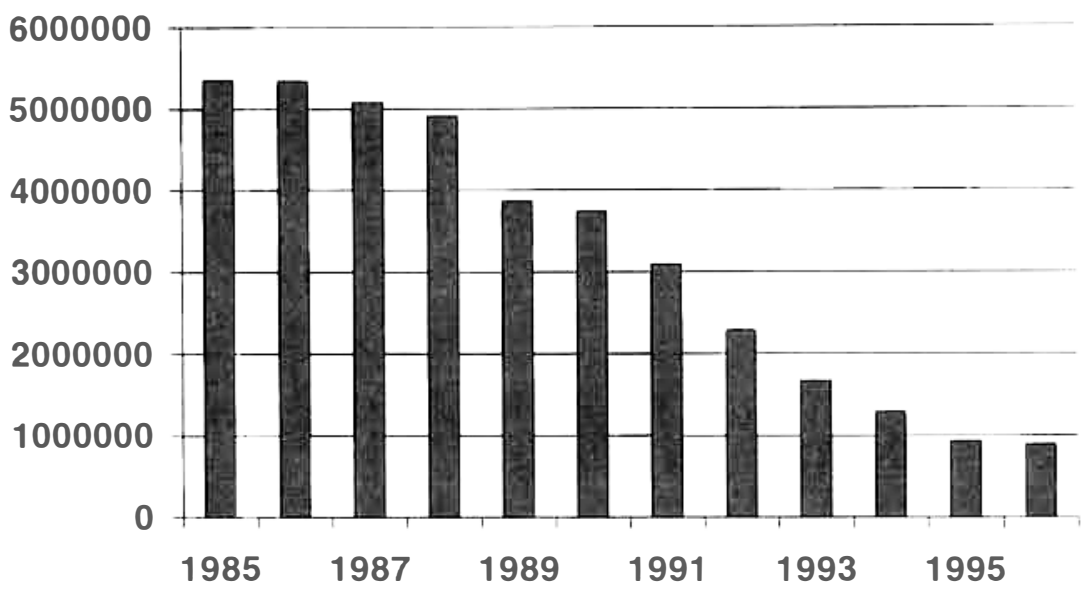

Figure 1. Global trend in registered cases, 1985-1996.

programme is well aware of this limitation and encourages national programme managers to estimate the true prevalence of leprosy each year based on guidelines ${ }^{3}$ for such estimations, these estimates are also regularly published. ${ }^{1}$ Over the past 10 years the gap between the estimated or true prevalence and the registered prevalence has narrowed and in 1997 it is estimated as around 260000 or about quarter of the true prevalence. Vigorous efforts are underway to register and treat these patients through Special Action Projects and the Leprosy Elimination Campaigns. It is therefore important to recognised that the registered prevalence of leprosy is not the same as the true prevalence.

\section{New case detection and the incidence of leprosy}

The incidence of a disease is the number of new cases which occur over a given period of time, usually per year. New case detection is considered as a measure which may reflect the incidence of leprosy however cases detected over the past year include patients who developed the disease many years previously. New cases may be registered in different centres at the same time duplicating the numbers of new cases reported or patients defaulting from treat may be re-registered as new cases (so called re-cycling of patients) artificially inflating the figures. Case detection figures are readily influenced by operational factors such as the intensity of cases detection methods, their frequency as well as by diagnostic changes. In contrast to the trends in leprosy prevalence which have fallen dramatically over the past 10 years, ${ }^{4}$ new case detection has remained remarkably unchanged over the same period (Figure 2). This global figure hides a range of different trends in case detection within individual countries (Figure 3). It is considered that these figures do not represent changes in the true incidence in these countries but reflect operational factors such as the expansion of geographical coverage by programmes, and intensification of case detection activities. It is therefore clear that not only are case detection figures a poor reflection of the true incidence of leprosy but also they are misleading. The constancy of the case detection figures over the past 


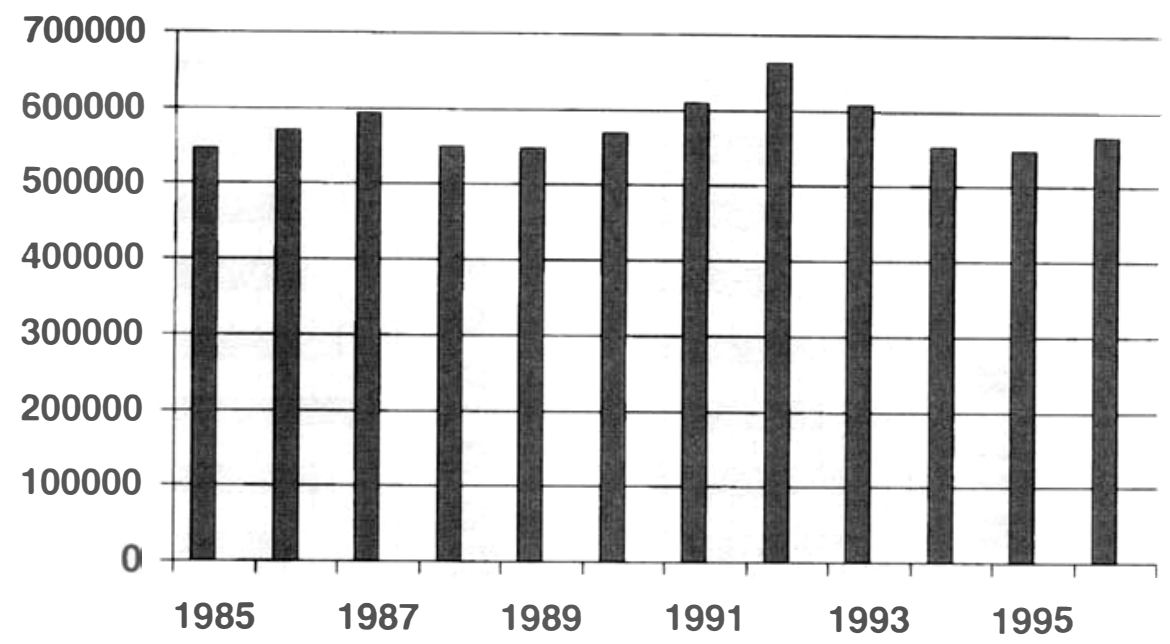

Figure 2. Global trend in new case detection, 1985-1996.

10 years at around 500-600 000 each year does not indicate a failure of the current elimination strategy but rather indicates its success in detecting and treating large numbers of previously undetected and untreated patients, the so called backlog cases. Similarly, any dramatic decline in new case detection in the next few years does not mean a sudden fall in disease incidence. It has been considered that the true incidence may be no more that a third of the annual case detection rate, this is a very rough estimate and is likely to vary greatly between individual countries. The current case detection figures do not tell us very much about the incidence of the disease.

\section{Incidence of disability and impairment could be more relevant}

It is often said that it is the disabilities which result from leprosy which set it apart from other diseases, both in terms of the primary impairments and the impairments secondary to nerve function loss. Disabilities or impairments could therefore be viewed as a relevant measure of the effect of anti-leprosy activities ${ }^{5}$ although communities attitudes can handicap individuals who have no nerve function impairments. A single skin lesion may be of little or no consequence and the detection of increased numbers of such cases can greatly inflate case detection figures while making the percentage with disability at detection appear artificially low. It can be argued that the incidence of disability or impairment is a more relevant measure, counting the absolute numbers of patients with WHO grade 2 disability at detection and any who develop new, irreversible nerve impairments during or immediately after MDT. The global number of new cases with such disability at detection is shown in Figure 4, however the pattern varies between countries. A global target of aiming at zero disability incidence could be developed, it would be a target relevant to patients as well as easily measured. A fall in new case detection with an increase in disability would not be a real benefit to patients, their families nor their communities. 

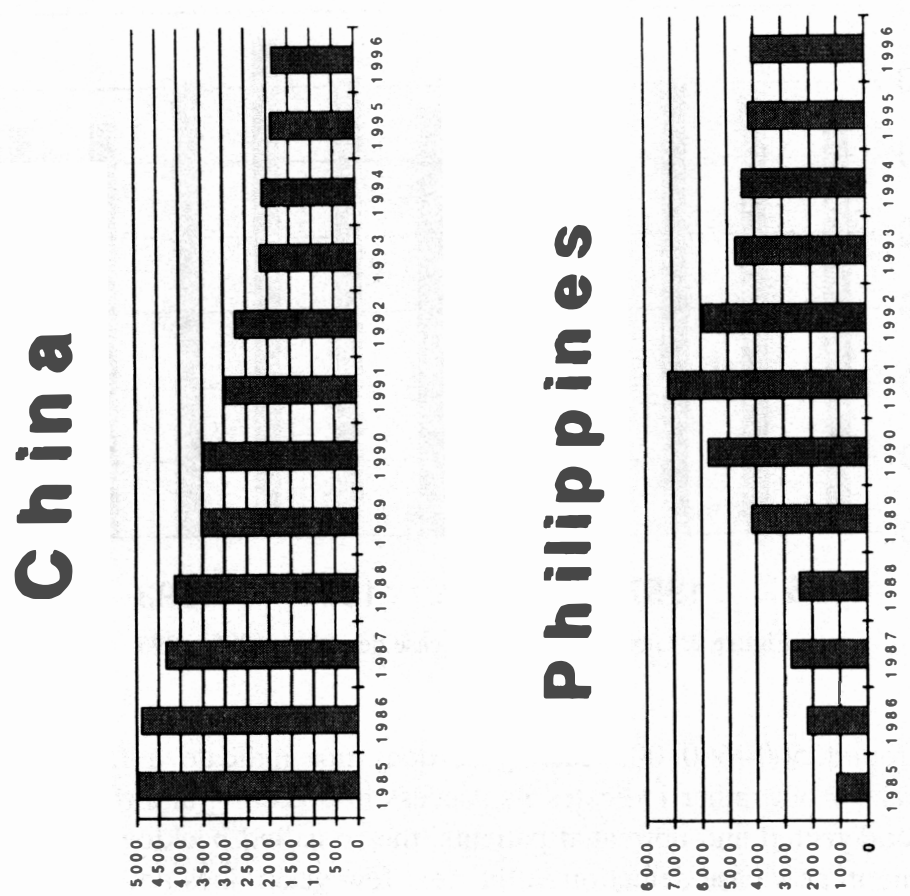

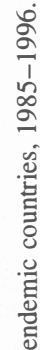

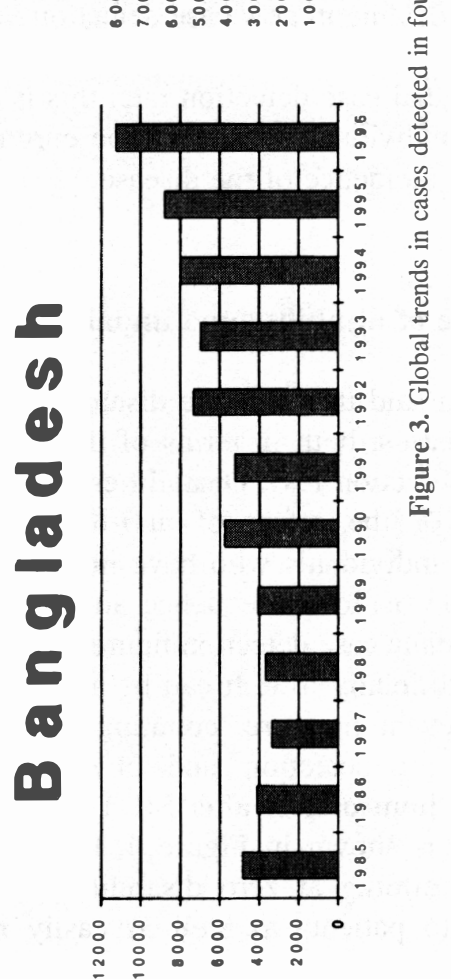




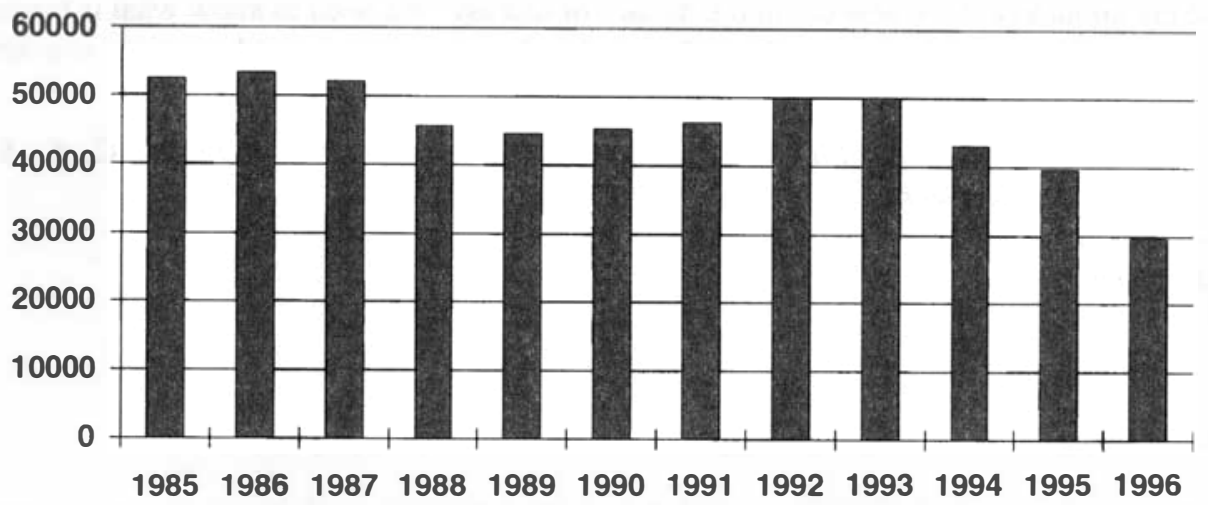

Figure 4. Global trend in new cases with disabilities, 1985-1996.

\section{How can we find out what is happening to the incidence of leprosy}

The collection of routine data on leprosy which is currently being undertaken, while necessary to monitor the elimination programme, does not tell us much about the incidence of leprosy in the main endemic countries. Special monitoring studies are needed to assess the incidence of leprosy as well as the application of new technologies and the use of simulation models to facilitate better understanding of what is going on.

Special studies are needed in population based programmes within the major endemic countries to monitor new case detection based on standardized case detection methods and consistent diagnostic criteria over many years. Collaborative studies which standardise methods and co-ordinate data collection in a number of populations in the endemic countries would begin to provide the data which are urgently required. These could start with retrospective analyses followed by the setting up of prospective monitoring. This is technically feasible as similar approaches have had to be developed for new case monitoring in the vaccine trials.

The development of the polymerase chain reaction (PCR) technology which has now been successfully applied in leprosy ${ }^{6}$ has potential for tracking transmission and incidence of infection as well as disease. Studies are being set up, funded by the European Commission (MILEP2), to evaluate this approach in Africa and Asia. More work is needed in this field. The development of simulation models such as SIMLEP and their application using different data sets can be used to study the relative importance of different modes of transmission and other transmission parameters, and analyse the potential impact of different intervention strategies working towards leprosy eradication as opposed to elimination.

\section{The priority for action}

It is perhaps surprising how little we know about the transmission and incidence of leprosy. We do not even know what the current incidence of leprosy is, or its trend in the major endemic countries. The prevalence of leprosy has dramatically fallen following the widespread implementation of MDT. The target prevalence rate of 1 in 10000 is approaching and 
we are about to enter a new era in the history of leprosy. We need to know what is happening to the incidence of leprosy to be able to plan for the next era and to work towards the eradication of leprosy.

Department of Public Health,

Dr W. C. S. SMith

University of Aberdeen,

Foresterhill,

Aberdeen

AB9 2ZD

\section{References}

${ }^{1}$ World Health Organization. Progress towards leprosy elimination. Weekly Epidemiological Record, 1997; 72: 165-172.

2 Irgens LM, Skjaerven R. Secular trends in age t onset, sex ratio, and type index in leprosy observed during declining incidence rates. Am J Epidemiol, 1985; 122: 695-705.

3 World Health Organization. Guidelines for Leprosy Elimination, 1996.

${ }^{4}$ World Health Organization. Global case-detection trend in leprosy. Weekly Epidemiological Record, 1997; 72: 173-180.

5 Smith WCS, Parkhe SM. Disability as relevant measure of progress in leprosy control. Lepr Rev, 1986; 57: 251-259.

${ }^{6}$ Hatta M, van Beers SM, Madjid B, Djumadi A, de Wit MYL, Klaster PR. Spatial distribution and persistance of Mycobacterium leprae nasal carriage among a population in which leprosy is endemic in Indonesia. Trans $R$ Soc Trop Med Hyg, 1995; 89: 381-385. 